\title{
VOLUME XVII
}

\section{AUTHOR INDEX}

\section{Papers and Technical Notes}

Short papers and notes are marked (N) in this index

Author

Batten, George W., Jr.

Bellman, Richard,

Kalaba, Robert, \&

Kotrin, Bella

Bramble, James H.

Brillhart, JOHN

Burgoyne, F. D.

Burgoyne, F. D.

Burgoyne, F. D.

Cavior, S. R.

Concus, P., Cassatt, D., JAEHNig, G., \& MELBY, E.

DaLy, Bart J.

DAY, J. T.

Descloux, J.

Douglas, Jim, JR., \& Gunn, James E.

Douglas, J., JR., KelLOGG, R. B. \& VARGA, R. S.

Erber, Thomas \& GorDon, Alan

Franklin, Joel N.

Ginsberg, F.

\section{Golub, Gene H}

Grace, Donald W. Johnson, Selmer M.

Keller, H. B. \& SwenSON, J. R.

LaBudde, C. Donald

LUKE, Y. L. \& WIMP, J.

Mapes, David C.
Title

Seond-order correct boundary conditions for the numerical solution of the mixed boundary problem for parabolic

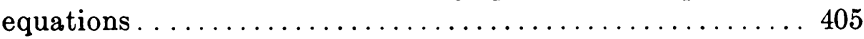

Polynomial approximation-a new computational technique in dynamic programming: allocation processes

Fourth-order finite difference analogues of the Dirichlet problem for Poisson's equation in three and four dimensions . . 217

Some miscellaneous factorizations $(\mathrm{N}) \ldots \ldots \ldots \ldots \ldots \ldots 47$ The non-existence of certain Laguerre-Chebyshev quadrature

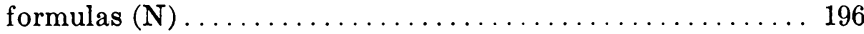

Approximations to Kelvin functions (N) . . . . . . . . . 295

Quadrature formulas over infinite intervals in terms of differ-

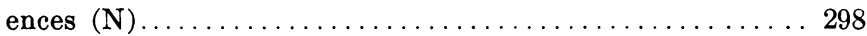

A note on octic permutation polynomials $(\mathrm{N}) \ldots \ldots \ldots \ldots 450$

Tables for the evaluation of $\int_{0}^{\infty} x^{\beta} e^{-x} f(x) d x$ by Gauss-Laguerre

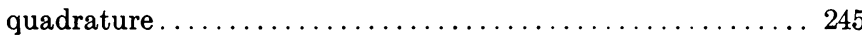

The stability properties of a coupled pair of non-linear partial difference equations . . . . . . . . . . . . . . . . . . . 346

A Gaussian quadrature method for the numerical solution of the characteristic initial value problem $u_{x y}=f(x, y, u)(\mathrm{N}) .438$

Note on the round-off errors in iterative processes........ 18

Two high-order correct difference analogues for the equation of multidimensional heat flow ................ 71

Alternating direction iteration methods for $n$ space variables

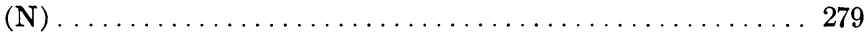

Tabulation of the Functions $\frac{\partial I_{\nu}(2)}{\partial \nu}, \nu= \pm \frac{1}{3} \ldots \ldots \ldots \ldots 2$

Deterministic simulation of random processes......... 28

On the Cauchy problem for the one-dimensional heat equa-

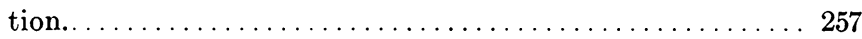

On a lower bound for the rank of a partitioned square matrix

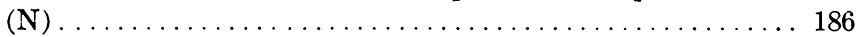

Search for largest polyhedra $(\mathrm{N}) \ldots \ldots \ldots \ldots \ldots \ldots \ldots \ldots \ldots$

Generation of permutations by adjacent transposition (N) . . 282

Experiments on the lattice problem of Gauss............ 223

The reduction of an arbitrary real square matrix to tri-diagonal form using similarity transformations $(\mathrm{N}) \ldots \ldots \ldots \ldots \ldots 43$

Jacobi polynomial expansions of a generalized hypergeometric

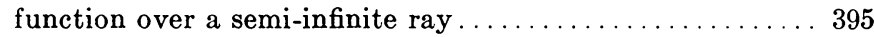

Fast method for computing the number of primes less than a given limit 
Author

MCANDREw, M. H.

McLaren, A. D.

Michels, H. H.

Miller, JAMes

Mrtchell, A. R. \& Pearce, R. P.

Navot, Israel

Newbery, A. C. R.

Newman, J. N. \&

Frank, W.

Pearson, Erna H.

Petryshyn, W. V.

A partition problem $(\mathrm{N}) \ldots \ldots \ldots \ldots \ldots \ldots \ldots \ldots \ldots \ldots . \ldots 291$

Optimal numerical integration on a sphere .............. 361

Abscissas and weight coefficients for Lobatto quadrature.... . 237

Formulas for integrals of products of associated Legendre or Laguerre functions $(\mathrm{N}) \ldots \ldots \ldots \ldots \ldots \ldots \ldots \ldots \ldots \ldots . \ldots \ldots$

Explicit difference methods for solving the cylindrical heat conduction equation .......................... 426

The Euler-Maclaurin functional for functions with a quasistep discontinuity ............................ 337

Multistep integration formulas $(\mathrm{N}) \ldots \ldots \ldots \ldots \ldots \ldots \ldots 452$

An integral containing the square of a Bessel function..... 64

On the congruences $(p-1) ! \equiv-1$ and $2^{p-1} \equiv 1\left(\bmod p^{2}\right)(\mathrm{N}) \ldots 194$

On a general iterative method for the approximate solution of linear operator equations ...................... 1

Polachek, H., Walton, Transient motion of an elastic cable immersed in a fluid.... 60 T. S., MEJIA, R., \&

Dawson, C.

Porsching, T. A.

Matrix assignments and an associated min max problem $(\mathrm{N}) \ldots \quad 81$

Preisendorfer, R. W. \& On the best fit to experimental data curves using homogeneous Roos, B. W. two-parameter functions....................... 231

Preisendorfer, R. W., Determinants by means of solutions of linear equations (N) . 285 Roos, B. W. \& SANGREN, W. C.

Riesel, HaNS

A factor of the Fermat number $F_{19}(\mathrm{~N}) \ldots \ldots \ldots \ldots \ldots \ldots 45$

Sankar, R. \& Malini, V. A note on the relative merits of Padé and Maehly's diagonal convergents in computing $e^{x} \ldots \ldots \ldots \ldots \ldots \ldots \ldots \ldots . \ldots \ldots$

Schinzel, A.

A remark on a paper of Bateman and Horn $(\mathrm{N}) \ldots \ldots \ldots . . \ldots 45$

Shanks, Daniel

Shanks, Daniel \&

Supplementary data and remarks concerning a Hardy-Littlewood conjecture $(\mathrm{N}) \ldots \ldots \ldots \ldots \ldots \ldots \ldots \ldots$

WRENCH, JoHN W., Jr.

Shapiro, George

The calculation of certain Dirichlet series............ 136

Stancu, D. D.

Gauss elimination for singular matrices $(\mathrm{N}) \ldots \ldots \ldots \ldots . \ldots 41$

Evaluation of the remainder term in approximation formulas by Bernstein polynomials.................... 270

Stancu, D. D. \& Stroud, Quadrature formulas with simple Gaussian nodes and multiple

A. H.

fixed nodes............................... 384

Stroud, A. H. \& Secrest, Approximate integration formulas for certain spherically Don

Stroud, A. H.

Sweeney, Dura W.

von HoLdT, R. E. \& symmetric regions ............................. 105

Coefficients in quadrature formulas $(\mathrm{N}) \ldots \ldots \ldots \ldots \ldots \ldots 289$

On the computation of Euler's constant.............. 170

HOWERTON, R. J.

Werner, H. \& Raymann, An approximation to the Fermi integral $F_{1 / 2}(x)(\mathrm{N}) \ldots \ldots \ldots 193$ G.

Whittlesey, John R. B. Incomplete Gamma functions for evaluating Erlang process probabilities................................. 11

Wilf, Herbert S.

Calculations relating to a conjecture of Pólya and Schoen-

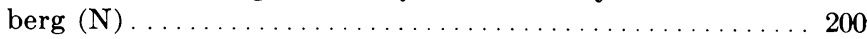

WUNDERLich, M.

On the non-existence of Fibonacci squares $(\mathrm{N}) \ldots \ldots \ldots \ldots 45$ 


\section{INDEX OF REVIEWS BY AUTHOR OF WORK REVIEWED}

\section{Author}

Ackoff, R. L.

Aizenshtat, V. S.

Allen, William A.

Alt, Franz L., Editor

ANCKer, C. J., JR.

A.NdReE, Richard V.

BAILEY, C. B.

Bartee, Thomas C.

Bell, Alan

Belousov, S. L.

Bennett, B. M.

Berlyand, O. S.

Bernard, Gary D.

Birkhoff, G.

Brown, B. M.

Carmichael, Robert D.

Centre Belge de Recherches MatheMATIQUES

Clenshaw, C. W.

Comrie, L. J.

Conte, Samuel D.

Coulson, John E.

Davis, H. T.

Dettman, John W.

Duffin, John H.

Durand, Émile

Fisher, Vera J.

Fletcher, A.

Ford, L. R., JR.

Fowler, F. P., Jr.

Frevel, L. K.

Fried, Burton D.

Fulkerson, D. R.

Fuller, L. E.

Gafarian, A. V.

Galler, Bernard A.

Gavrilova, R. I.

Gel'fand, I. M.

GlembockiJ, J.

Glinski, Anna

Govindarajulu, ZakKula

Greenberger, Martin, Editor

Gupta, S. K.

Hahn, Wolfgang

Hall, J. A. P., Editor

Hanson, Donald N.

Hartley, G. M.

Herrera, Lee

Higgens, T. P.

Higgins, Adele

Hilsenrath, Joseph

\author{
Review \\ Number
}

47

9

24

15

22

72

69

92

71

79

45

80

49

85

5

70

28

1

33

10

61

68

82

56

16

68

33

58

12

81

10

58

42

22

93

80

74

26

19

20

14

47

87

62

56

94

76

11

71

53
Table

Classification

Page

[K, W]

[L]

[L]

[X, Z]

[L, M]

$\mathrm{F}$

[A, B]

[Z]

$[\mathrm{A}, \mathrm{I}]$

[L]

[K]

[L]

[L]

[S, X]

[I, K, P]

[A-E, J, M]

[X]

313

93

207

98

206

463

461

481

462

470

311

470

315

475

90

461

209

[C, D, E, L, X] 88

[A-E, G-N, X, Z] 302

[L] $\quad 94$

[Z] 327

[A, B] 459

$[\mathrm{P}, \mathrm{S}, \mathrm{X}] \quad 472$

[T, Z] 323

[G, H] 202

[A, B] 459

[A-E, G-N, X, Z] 302

[W] 325

[W] 95

[L] 471

[L] 94

[W] 325

[G] 309

[L, M] 206

[Z] 482

[L] 470

[G] 464

[M, S] 208

[K] 203

[K] 204

[W, Z] 97

[K, W] 313

[X] 477

[Z] $\quad 328$

[T, Z] 323

[Z] 483

[K] 466

[L] 95

[A, I] 462

$[\mathrm{L}, \mathrm{S}]$ 


\begin{tabular}{|c|c|c|c|}
\hline Author & $\begin{array}{l}\text { Review } \\
\text { Number }\end{array}$ & $\begin{array}{c}\text { Table } \\
\text { Classification }\end{array}$ & Page \\
\hline Hodgman, Charles D., Editor & 34 & {$[\mathrm{~A}-\mathrm{F}, \mathrm{K}-\mathrm{N}, \mathrm{Q}]$} & 303 \\
\hline Hollitch, Robert S. & 86 & {$[\mathrm{~W}, \mathrm{Z}]$} & 476 \\
\hline IsHIMARU, AKIRA & 49 & {$[\mathrm{~L}]$} & 315 \\
\hline $\mathrm{J}_{\mathrm{ACOB}}, \mathrm{C}_{\text {AIUS }}$ & 57 & [V] & 324 \\
\hline JEFFREYS, HAROLD & 18 & {$[\mathrm{~J}, \mathrm{~L}, \mathrm{M}]$} & 203 \\
\hline KAGLE, B. J. & 50 & [L] & 317 \\
\hline KaPLAN, WILFRED & 23 & {$[\mathrm{~L}, \mathrm{M}, \mathrm{X}]$} & 206 \\
\hline KARPLUS, WALTER J. & 66 & [Z] & 331 \\
\hline Karush, William & 88 & {$[\mathrm{X}]$} & 478 \\
\hline Kimbro, Genevieve M. & 17 & {$[\mathrm{I}]$} & 202 \\
\hline KNOLLE, WeRner E. & 24 & {$[\mathrm{~L}]$} & 207 \\
\hline KRASOvSKII, N. N. & 89 & {$[\mathrm{X}]$} & 478 \\
\hline KRYLOV, V. I. & 9 & [L] & 93 \\
\hline LEBOW, IRWIN L. & 92 & [Z] & 481 \\
\hline LedLey, Robert S. & 63 & {$[Z]$} & 329 \\
\hline Levine, Norman & 35 & [D] & 304 \\
\hline LUKE, YUdELL L. & 51 & {$[\mathrm{~L}, \mathrm{M}]$} & 318 \\
\hline Mainland, Donald & 76 & {$[\mathrm{~K}]$} & 466 \\
\hline MAPES, DAvid C. & 39 & {$[\mathrm{~F}]$} & 307 \\
\hline Marcus, Mitchell P. & 27 & {$[\mathrm{P}, \mathrm{Z}]$} & 208 \\
\hline MASKET, A. V. H. & 25 & {$[\mathrm{~L}, \mathrm{M}]$} & 207 \\
\hline McCracken, Daniel D. & 29 & {$[\mathrm{Z}]$} & 210 \\
\hline McCracken, Daniel D. & 64 & [Z] & 330 \\
\hline MCKeE, Lowry & 72 & {$[\mathrm{~F}]$} & 463 \\
\hline MetLeskit, A. S. & 9 & {$[\mathrm{~L}]$} & 93 \\
\hline MiLLeR, J. C. P. & 2 & {$[\mathrm{~F}]$} & 88 \\
\hline Miller, J. C. P. & 33 & {$[\mathrm{~A}-\mathrm{E}, \mathrm{G}-\mathrm{N}, \mathrm{X}, \mathrm{Z}]$} & 302 \\
\hline Minas, J. S. & 47 & {$[\mathrm{~K}, \mathrm{~W}]$} & 313 \\
\hline Mitrinović, D. S. & 6 & {$[\mathrm{I}, \mathrm{X}]$} & 90 \\
\hline Mitrinović, D. S. & 44 & [I] & 311 \\
\hline Mitrinović, R. S. & 6 & {$[\mathrm{I}, \mathrm{X}]$} & 90 \\
\hline Mitrinović, R. S. & 44 & [I] & 311 \\
\hline Mittman, Benjamin & 86 & {$[\mathrm{~W}, \mathrm{Z}]$} & 476 \\
\hline Montpetit, G. & 67 & {$[\mathrm{~A}]$} & 459 \\
\hline NAKAMURA, E. & 45 & [K] & 311 \\
\hline Nichol, C. А. & 72 & {$[F]$} & 463 \\
\hline Oberhettinger, Fritz & 11 & [L] & 95 \\
\hline Olver, F. W. J. & 75 & {$[\mathrm{I}, \mathrm{L}]$} & 464 \\
\hline Papoulis, Athanasios & 55 & {$[\mathrm{M}, \mathrm{X}]$} & 322 \\
\hline Paran, E. & 50 & {$[\mathrm{~L}]$} & 317 \\
\hline Peterson, E. L. & 8 & {$[\mathrm{~K}]$} & 92 \\
\hline Plette, William S. & 65 & {$[\mathrm{Z}]$} & 331 \\
\hline Pontryagin, L. S. & 54 & {$[\mathrm{M}]$} & 322 \\
\hline Prudnikov, A. P. & 80 & {$[\mathrm{~L}]$} & 470 \\
\hline ReEd, Irving S. & 92 & {$[\mathrm{Z}]$} & 481 \\
\hline REID, J. B. & 67 & {$[\mathrm{~A}]$} & 459 \\
\hline REIs, G. E. & 69 & {$[\mathrm{~A}, \mathrm{~B}]$} & 461 \\
\hline $\begin{array}{l}\text { Rheinisch-Westfälisches Institut für } \\
\text { Instrumentelle Mathematik }\end{array}$ & 30 & {$[\mathrm{Z}]$} & 210 \\
\hline RODGERS, W. C. & 25 & {$[\mathrm{~L}, \mathrm{M}]$} & 207 \\
\hline ROSENHEAD, L. & 33 & {$[\mathrm{~A}-\mathrm{E}, \mathrm{G}-\mathrm{N}, \mathrm{X}, \mathrm{Z}]$} & 302 \\
\hline ROSSER, J. BARKLEY & 40 & {$[\mathrm{~F}]$} & 307 \\
\hline Salzer, Herbert E. & 17 & {$[\mathrm{I}]$} & 202 \\
\hline Salzer, Herbert E. & 35 & [D] & 304 \\
\hline
\end{tabular}


Author

Saxon, James A.

Scarborough, James B.

eview

Table

Schoenfeld, Lowell

Classification

Page

65

[Z]

331

90

[X]

[F]

479

307

SchwatT, I. J.

[J] 91

Sebestyen, George S.

Selby, Samuel M., Editor

$[\mathrm{X}, \mathrm{Z}]$

479

[A-F, K-N, Q] 303

Selfridge, John L.

[F] 463

Shankland, Robert S., Editor 34

Shanks, DaNiEl

[A-F, K-N, Q] 303

Smith, Edwin R.

Somerville, Graham F.

[F]

464

[A-E, J, M] 461

SpICER, H. C.

SpICER, H. C.

$[\mathrm{T}, \mathrm{Z}]$

461
323

[E] 305

[E] 306

Spicer, H. C.

SpIra, Robert

Stuart, R. D.

[L, M]

306

[F] 306

$[\mathrm{P}, \mathrm{X}] \quad 473$

Sutcliffe, Marion I.

[K]

Szegö, Gabor, et al, Editors

$[\mathrm{F}, \mathrm{G}, \mathrm{X}]$

466 .

Teodorescu, P. P.

Thacher, Henry C., Jr.

$[\mathrm{P}, \mathrm{X}]$

308

[I] 202

Thorn, MarJory M.

$[\mathrm{F}, \mathrm{G}, \mathrm{I}, \mathrm{X}] \quad 89$

TodD, John, Editor

[Z]

[L]

331

Turley, J. W.

UŠPALIS, K.

VANAGas, V.

VAN DYKE, JOHN

VARGa, Richard S.

Ventzel, E. S.

Vichnevetsky, R., Editor

Wagner, Harvey $M$.

$[\mathrm{M}, \mathrm{S}]$

471

208

[K] 203

$[\mathrm{G}, \mathrm{H}, \mathrm{X}] \quad 310$

[W] 97

[Z] 211

$[\mathrm{K}, \mathrm{W}]$

Weast, Robert C., Editor

[A-F, K-N, Q] 303

Western Data Processing Center, Uni-

[W]

Wigner, E. P., Editor

$[\mathrm{S}, \mathrm{X}]$

Wilks, Samuel $\mathrm{S}$.

[K]

Williams, R. W.

Wolf, Frank L.

YAGLOM, A. M.

Zaludova, A. H.

Ziegler, Guy G.

ZIPPERER, I.

[Z]

$[\mathrm{K}]$

$[\mathrm{K}]$

[K]

$[\mathrm{L}, \mathrm{S}]$

[X]

\section{NOTE}

Title

Page

Translation of Russian Journal

\section{TABLE ERRATA}

No.

Author

Title

337 Admiralty Computing Tables of Legendre Functions $Q_{n}(x)$. 


\author{
No. \\ Author \\ 323 Allen, E. S. \\ 320 Bohlin, K. \\ 329 Bruhns, C. C. \\ 334 Croarkin, Mary C. \\ 328 DALE, J. B.
}

338 Tricomi, F. G. L., \& Comrie, L. J. Sliepcerich, C. M. HAYASHI, K.

\section{JAHNKE-EMIDE-LÖSCH}

Lanczos, C.

LÉvy, L.

Milne, W. E.

Riesel, H. STEIN, I. S.

Silberstein, L.

Slater, L. J.

Spenceley, G. W. \&

Spenceley, G. W.,

Epperson, E. R.

SPenceley, G. W.,
Erdéltyi, A., Magnus, W. OberhetTinger, F. \&

Fletcher, A., Miller, An Index of Mathematical Tables, Vol. I and II. J. C. P., Rosenhead,

Gumprecht, R. O. \&

HANCOCK, HARRIS

Lowan, A. N., Salzer, H. E., \& Hillman, A.

Ryshik, I. M. \& GradSPENCELey, R. M. SPENCELEy, R. M. \&

Spenceley, R. M. \&

EPperson, E. R.

Tables of Riccati Bessel Functions for Large Arguments and

Tafeln für die Differenzenrechnung sowie für die Hyperbel-,

Précis Elémentaire de la Théorie des Fonctions Elliptiques

Numerical Calcultrs........................... 214

"All factors $q<10^{8}$ in all Mersenne numbers $2^{p-1}, p$ prime

Smithsonian Logarithmic Tables to Base e and Base 10 . . 103

Smithsonian Logarithmic Tables to Base e and Base 10... 215

Title

Page

Six-Place Tables.............................. 101

Tables des Fonctions Elliptiques. .................. 100

A New Manual of Logarithms to Seven Places of Decimals. . 214

"Graphs for determining the power of Student's $t$-test". . 334

Five-Figure Tables of Mathematical Functions Comprising Tables of Logarithms, Powers of Numbers, Trigonometric, Elliptic and other Transcendental Functions. . 103

Higher Transcendental Functions. . . . . . . . . . . . . . . 485

Orders.................................... 102

Elliptic Integrals.......................... 100 Besselschen, elliptischen und anderen Funktionen. . . . 102

Tables of Higher Functions. . . . . . . . . . . . . . . . . 485

Applied Analysis ............................ 334 avec Tables Numériques et Applications............. 100

"A table of coefficients for numerical differentiation"... 334

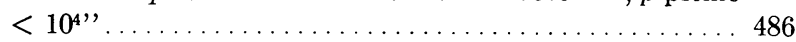

Summen-, produkt- und Integral-Tafeln............ 102

Synopsis of Applicable Mathematics............... 101

Confluent Hypergeometric Functions.................. 486

Smithsonian Elliptic Functions Tables.............. 214

CORRIGENDA

Author

Alt, Franz L., Editor

Bramble, James $\mathrm{H}$.

Brillhart, John

BRillhart, JohN

Brillhart, John \&

Johnson, G. D.

Carlitz, L.

Fraser, W., \& Gotlieb, C. C.

Grace, Donald W.
Title

Page

Advances in Computers......................... 335

"Fourth-order finite difference analogues of the Dirichlet prob-

lem for Poisson's equation in three and four dimensions". . 487

"Concerning the numbers $2^{2 p}+1, p$ prime" $\ldots \ldots \ldots \ldots \ldots 215$

"Concerning the numbers $2^{2 p}+1, p$ prime" ............. 487

"On the factors of certain Mersenne numbers"........... 215

"The coefficients of the leminiscate function" ......... 215

"A calculation of the number of lattice points in the circle and sphere" .................................. 104

"Search for largest polyhedra" . . . . . . . . . . . . . . 488 
Hansen, Eldon R. \& Patrick, Merrell L.

Ralston, Anthony

Shanks, Daniel \& WRENCH, JoHN W., JR.

Sweeney, Dura W.
"Some relations and values for the generalized Riemann Zeta function" .................................. 104

"Runge-Kutta methods with minimum error bounds" .... . 488

"The calculation of certain Dirichlet series" ............488

"On the computation of Euler's constant" ............4488

\section{REVIEWS BY SUBJECT}

\section{A. Arithmetical Tables, Mathematical Constants}

\footnotetext{
Author/s

Bailey, C. B. \& Reis, G. E.

Beli, Alan \& Higgins, Adele

Carmichael, Robert D. \& Smith, Edwin R.

Davis, H. T. \& Fisher, VERA J.

Fletcher, A., Miller, J. C. P., Rosenhead, L. \& Comrie, L. J.

Reid, J. B., \& Montpetit, G.

Selby Samuel, M., Weast, Robert C., Shankland, Robert S., \& Hodgman, Charles D., Editors
}

Bailey, C. B. \& Reis, G. E.

Carmichael, Robert D. \& Smith, Edwin R.

Davis, H. T. \& Fisher, VERA J.

Fletcher, A., Miller, J. C. P., Rosenhead, L. \& Comrie, L. J.

Selby, Samuel M., Weast, Robert C., Shankland, Robert S. \& Hodgman, Charles D., Editors

Carmichael, Robert D. \& SMITH, EDwin R.

Clenshaw, C. W

Fuenchar, A., MiLLER, J. C. P., Rosenhead, L., \& Comrie, L. J.

Review Title Page

69 Tables of Roots of the First Ten Thousand Integers. . 461

71 Table of Stirling Numbers of the Second Kind $S(n, k)$, $k=1(1) n, n=1(1) 100 \ldots \ldots \ldots \ldots \ldots \ldots \ldots 4 . \ldots \ldots 2$

$70 \quad$ Mathematical Tables and Formulas............. 461

68 Tables of the Mathematical Functions, Arithmetical Tables, Volume III ...................... 459

An Index of Mathematical Tables, Second edition. . . 302

\section{B. Powers}

\section{Logarithms}

Mathematical Tables and Formulas.............461

Chebyshev Series for Mathematical Functions...... S\$ An Index of Mathematical Tables.............. 302 


\footnotetext{
Author/s

Selby, Samuel M., Weast, Robert C., ShankLand, Robert S. \& Hodgman, Charles D., Editors
}

Carmichael, Robert D. \& Smith, Edwin R.

Clenshaw, C. W.

Fletcher, A., Miller, J. C. P., Rosenhead, L. \& Comrie, L. J.

Salzer, Herbert E. \& Levine, Norman

Selby, Samuel M., Weast, Robert C., Shankland, Robert S. \& Hodgman, Charles D., Editors
Review

\section{Circular Functions}

70

Mathematical Tables and Formulas. 461

1 Chebyshev Series for Mathematical Functions....... 88

33 An Index of Mathematical Tables, Second edition . . 302

Table of Sines and Cosines to Ten Decimal Places at Thousandths of a Degree. .................. 304

$34 \quad$ Handbook of Mathematical Tables.............. 303

\section{E. Hyperbolic and Exponential Functions}

Carmichael, Robert D. \& Smith, Edwin R.

Clenshaw, C. W.

Fletcher, A., Miller, J. C. P., Rosenhead, L. \& Comrie, L. J.

Selby, Samuel M. Weast, Robert C., ShankLand, Robert S. \& Hodgman, Charles D., Editors

SPICER, H. C.

Spicer, H. C.

Mapes, David C.

Miller, J. C. P.

Nichol, C. A., Selfridge, JOHN L. MCKEe, LOWRY \& RICHARD V. ANDREE

Rosser, J. BARKLey \& SCHOENFELD, LOWELI

Selby, Samuel M., Weast, Robert C., Shankland, Robert S. \& Hodgman, Charles D., Editors

Shanks, Daniel

SpIra, Robert
70

Mathematical Tables and Formulas. 461

Chebyshev Series for Mathematical Functions...... 88

33

An Index of Mathematical Tables, Second edition . . 302

\section{F. Theory of Numbers}

Fast Method for Computing the Number of Primes less than a Given Limit.................... 307

Table of Least Primitive Roots................ 88 A Table of Indices and Power Residues for All Primes and Prime Powers Below 2000............. 463

40 "Approximate formulas for some functions of prime numbers"......................... 307

$34 \quad$ Handbook of Mathematical Tables.............. 303 
Author/s

Szegö, Gabor, et al, Editors

TodD, JoHn, Editor

Durand, Énile

Fletcher, A., Miller, J.C.P., Rosenhead, L. \& Comrie, L. J.

Fuller, L. E.

Gel'fand, I. M.

Szegö, Gabor, et al, Editors

Tond, John, Editor VARGA, Richard S.

Durand, Émile

Fletcher, A. Miller, J. C. P., Rosenhead, L. \& Comrie, L. J.

Thacher, Henry C., JR. VARGa, Richard S.

Bell, Alan \&

Higgins, Adele

Brown, B. M.

Fletcher, A., Miller, J. C. P., Rosenhead, L. \& Comrie, L. J.

Mitrinović, D. S. \& Mitrinović, R. S.

Mitrinović, D. S. \& Mitrinović, R. S. Olver, F. W. J.

Salzer, Herbert E., Kimbro, Genevieve M. \& Thorn, Marjory M.

Todd, JoHn, Editor

Carmichael, Robert D. \& Smith, Edwin R.

Fletcher, A. Miller, J. C. P., Rosenhead, L. \& Comrie, L. J.

JeFfreys, Harold SchwatT, I. J.
Review

Title

Page

Studies in Mathematical Analysis and Related Topics

-Essays in Honor of George Pólya. . . . . . . . . . 308

$3 \quad$ A Survey of Numerical A nalysis.............. 89

\section{G. Higher Algebra}

Solutions Numériques des Équations Algébriques. . 202
42

74

41

3

43

\section{H. Numerical Solution of Equations}

16 Solutions Numériques des Équations Algébriques. . 202

33 An Index of Mathematical Tables, Second edition . . . 302

$4 \quad$ Real Roots of the Equation $x \tan y+\tanh y=0 \ldots .90$

$43 \quad$ Matrix Iterative Analysis. . . . . . . . . . . . 310

\section{Finite Differences, Interpolation}

71

\section{J. Summation of Series}

Tables of Stirling Numbers of the Second Kind $S(n, k), k=1(1) n, n=1(1) 100 \ldots \ldots \ldots \ldots \ldots \ldots 2$ The Mathematical Theory of Linear Systems. . . . . . 90 An Index of Mathematical Tables, Second edition... 302

"Sur une classe de nombres se rattachant aux nombres de Stirling"'...................90

Tableaux d'une classe de nombres reliés aux nombres de Stirling..................... 311

Tables for Bessel Functions of Moderate or Large Orders.......................... 464

Tables for Complex Hyperosculatory Interpolation over a Cartesian Grid. .................. 202

A Survey of Numerical Analysis.............. 89

Mathematical Tables and Formulas............ 461

An Index of Mathematical Tables, Second edition. . 302

Asymptotic Approximations. . . . ........... 203 An Introduction to the Operations with Series . . . . . . 91 


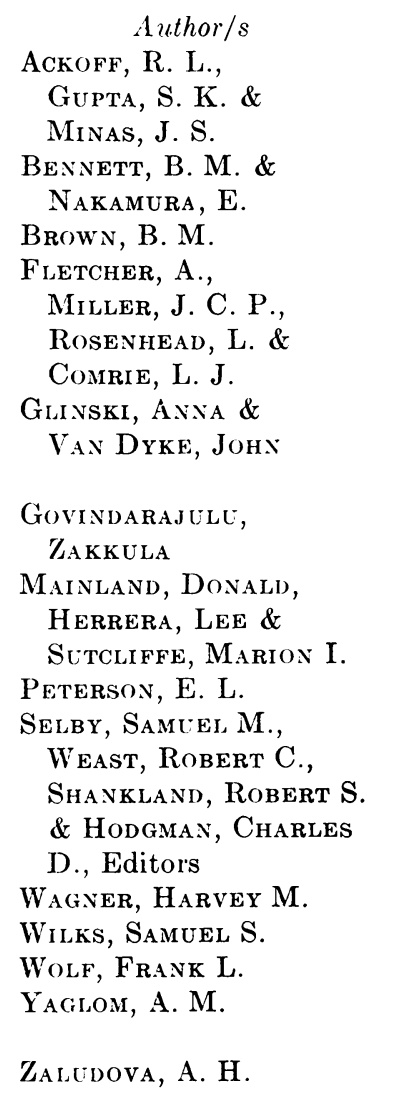

Review

Statistical Management of Inventory Systems. . . . . 314

Mathematical Statistics . . . . . . . . . . . . . . . 312

Elements of Probability and Statistics . . . . . . . . . 204 An Introduction to the Theory of Stationary Random

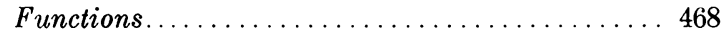

"The non-central $t$-test ( $q$-test) based on range in place of standard deviation" . . . . . . . .... 469

\section{Higher Mathematical Functions}

\section{Aizenshtat, V. S. Krylov, V. I. \& MetleskiI, A. S.}

Ancker, C. J., JR. \& Gafarian, A. V.

Belousov, S. L.

Berlyand, O. S., Gavrilova, R. I. \& Prudnikov, A. P. Ishimaru, Akira Clenshaw, C. W. Fletcher, A., Miller, J. C. P., Rosenhead, L. \& Comrie, L. J. Bernard, Gary D. \&
Tables for Calculating Laplace Transforms and Integrals of the form $\int_{0}^{\infty} x^{s} e^{x} f(x) d x \ldots \ldots \ldots \ldots \ldots$

22 79

$1 \quad$ Chebyshev Series for Mathematical Functions....... 88 33 An Index of Mathematical Tables, Second edition . . 302 ties and $a$ Table . . ...................... 206

Tables of Normalized Associated Legendre Polynomials...................... 470 Tables of Integral Error Functions and Hermite

Tables of the Anger and Lommel-Weber Functions. . 315 Polynomials.................... 470 
Author/s

Frevel, L. K. \&

Turley, J. W.

Fried, Burton D. \&

Conte, Samuel D.

Hilsenrath, Joseph \&

Ziegler, Guy G.

JEFFreys, Harold

Kaplan, Wilfred

Knolle, Werner E. \&

Allen, William A.

Luke, Yudell L.

Masket, A. V. H. \&

Rodgers, W. C.

Oberhettinger, Fritz \&

Higgens, T. P.

Olver, F. W. J.

Paran, E. \&

KaGLe, B. J.

Selby, Samuel M.,

Weast, Robert C.,

Shankland, Robert S

\& Hodgman, Charles

D., Editors

SpICER, H. C.
Review

81

10

53

18

23

24

51

25

11

75

50

34

52

AnCker, C. J., JR. \&

Gafarian, A. V.

Carmichael, Robert D. \& Smith, Edwin R.

Fretcher, A., Miller, J. C. P., Rosenhead, L. \& Comrie, L. J.

JefFreys, Harold

Kaplan, Wilfred

Luke, Yudell L.

Masket, A. V. H. \& Rodgers, W. C.

Papoulis, Athanasios

Pontryagin, L. S.

Selby, Samuel M., Weast, Robert C., Shankland, Robert S. \& Hodgman, Charles D., Editors
22

70

33

18

23

51

25

55

54

34

Title

Page

Tables of Iterated Bessel Functions of the First Kind

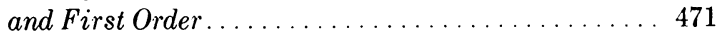

The Plasma Dispersion Function: The Hilbert Transform of the Gaussian. ............... 94

Tables of Einstein Functions-Vibrational Contributions to the Thermodynamic Functions. . . . . . . . 321

Asymptotic Approximations. . . . . . . . . . . 203

Operational Methods for Linear Systems . ......... 206

Expansion of Elliptic Functions Tables . . . . . . . 207

Integrals of Bessel Functions. . . . . . . . . . . 318

Tables of Solid Angles: I. Solid Angle Subtended by a Circular Disc; II. Solid Angle Subtended by the Lateral Surface of a Right Circular Cylinder. . . . . 207

Tables of Lebedev, Mehler, and Generalized Mehler Transforms....................... 95

Tables for Bessel Functions of Moderate or Large Orders............................. 464

Tables of Legendre Polynomials of the First and Second Kind. ..................... 317

Handbook of Mathematical Tables............. 303

Tables of the Inverse Probability Integral $P=\frac{2}{\sqrt{ } \pi} \int_{0}^{\beta} e^{-\beta^{2}} d \beta$

\section{Integrals}

The Function $J(x, y)=\int_{0}^{x} \frac{\gamma(y, \xi)}{\xi} d \xi$-Some Prop-

erties and $a$ Table.................... 206

Mathematical Tables and Formulas............. 461

An Index of Mathematical Tables, Second edition. . . 302

Asymptotic Approximations. . . . . . . . . . . . . 203

Operational Methods for Linear Systems . ......... 206

Integrals of Bessel Functions............... 318

Tables of Solid Angles: I. Solid Angle Subtended by a Circular Disc; II. Solid Angle Subtended by the Lateral Surface of a Right Circular Cylinder . . . . . 207

The Fourier Integral and its A pplications . . . . . . 322

Ordinary Differential Equations . . . . . . . . . . . . 322

Handbook of Mathematical Tables. . . . . . . . . . . 303 


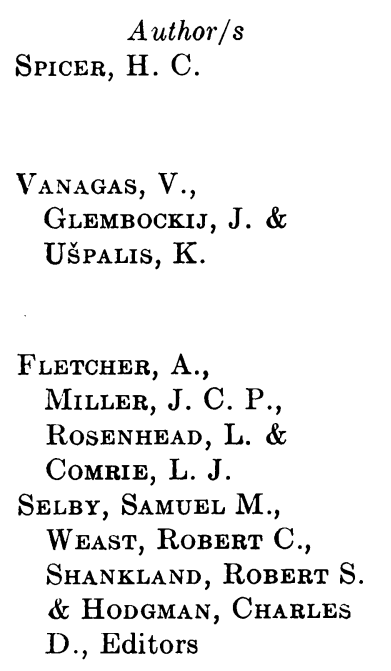

BRown, B. M.

Dettman, John W.

Marcus, Mitchell P.

Stuart, R. D.

Teodorescu, P. P.
Selby, Samuel M., Weast, Robert D., ShankLAND, Robert S. \& Hodgman, Charles D., Editors

Review

52

Tables of the Inverse Probability Integral

$$
P=\frac{2}{\sqrt{\pi}} \int_{0}^{\beta} e^{-\beta^{2}} d \beta .
$$

Tables of Radial Integrals of Atomic Spectra Theory.. 208

\section{N. Interest and Investment}

An Index of Mathematical Tables, Second Edition... 302

\section{P. Engineering}

The Mathematical Theory of Linear Systems....... . 90

82 Mathematical Methods in Physics and Engineering... 472

$27 \quad$ Switching Circuits for Engineers. . . . . . . . . . . . 208

$83 \quad$ An Introduction to Fourier Analysis . . . ........ 473

84 Probleme Plane in Teoria Elasticitatii, Vol. I...... 473

Q. Astronomy

\section{S. Physics, Geophysics, Crystallography}

Birkнoff, G. \& Wigner, E. P., Editors Dettman, John W. Hilsenrath, Joseph \& Ziegler, GuY G.

VANAGAS, V., GLembockiJ, J. \& UŠPALIS, $\mathrm{K}$.

Hanson, Donald N., DufFin, JoHN H. \& Somerville, Graham F.
85 Proceedings of Symposia in Applied Mathematics, Vol. XI, Nuclear Reactor Theory............. 475

82 Mathematical Methods in Physics and Engineering... 472

53 Tables of Einstein Functions-Vibrational Contributions to the Thermodynamic Functions.......... 321

26 Tables of Radial Integrals of Atomic Spectra Theory. 208

\section{T. Chemistry}

$56 \quad$ Computation of Multistage Separation Processes.... 323

\section{Aerodynamics, Hydrodynamics, Ballistics}

JaCOB, CaIUS

ACKOFF, R. L., Gupta, S. K., \& Mrnas, J. S.
$57 \quad$ Introduction Mathématique à la Méchanique des Fluides............................... 324

\section{W. Economics and Social Sciences}

47 Scientific Method: Optimizing Applied Research Decisions.............................. 313 
Author/s Review Title Page

FORD, L. R., JR. \&

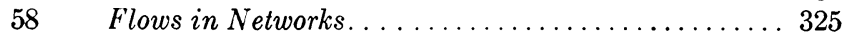

Fulkerson, D. R.

$\begin{array}{llll}\text { Fowler, F. P., JR. } & 12 & \text { Basic Mathematics for Administration........... } & 95 \\ \text { Greenberger, Martin, } & 14 & \text { Management and the Computer of the Future....... }\end{array}$ Editor

Hollitch, Robert S. \&

$86 \quad$ Computer Applications-1961 ........... 476

Mrttman, Benjamin

Ventzel, E. S.

$13 \quad$ Lectures on Game Theory................ 97

WAgNer, HARVEY M. $\quad 48 \quad$ Statistical Management of Inventory Systems...... 314

Western Data Process- $59 \quad$ Contributions to Scientific Research in Management. . 325 ing Center, UniverSity of California, Los ANGeles

X. Numerical Analysis and Applied Mathematics

Alt, Franz L., Editor

Birkhoff, G. \&

Wigner, E. P., Editors

Centre Belge de

ReCherChes

Mathématiques

Clenshaw, C. W.

Detrman, John W.

Fletcher, A.,

Miller, J. C. P.,

Rosenhead, L. \&

Comrie, L. J.

HaHn, WolfGang

Faplan, Willfred

Karush, William

Krasovskit, N. N.

Mitrinović, D. S. \&

Mitrinović, R. S.

Papoulis, Athanasios

Scarborough, James B.

Sebestyen, George S.

Stuart, R. D.

Szegö, Gabor, et al, Editors

Teodoresce, P. P.

Todd, John, Editor

Varga, Richard S.

Zi pperer, L.
15
Advances in Computers, Vol 2. Proceedings of Symposia in Applied Mathematics

Vol. XI, Nuclear Reactor Theory............. 475

Colloque sur l'A nalyse Numérique . . . . . . . . . . . 209

Chebyshev Series for Mathematical Functions...... 88

Mathematical Methods in Physics and Engineering... 472 An Index of Mathematical Tables, Second Edition... 302

Theory and Applications of Liapunov's Direct Method......................... 477

Operational Methods for Linear Systems . . . . . . . 206

The Crescent Dictionary of Mathematics . . . . . . . . 478

Stability of Motion................... 478

"Sur une classe de nombres se rattachant aux

nombres de Stirling" . ................ 90

The Fourier Integral and its Applications. . . . . . . 322

Numerical Mathematical Analysis. ............. 479

Decision-Making Processes in Pattern Recognition. 479

An Introduction to Fourier Analysis. . . . . . . . . 473

Studies in Mathematical Analysis and Related Topics

-Essays in Honor of George Pólya. . . . . . . . . . . 308

Probleme Plane in Teoria Elasticitatii, Vol. I. . . . . 473

A Survey of Numerical Analysis. . . . . . . . . . . . 89

Matrix Iterative Analysis. ............... 310

Tables for the Harmonic Analysis and Synthesis of Periodic Functions

\section{Z. Calculating Machines and Mechanical Computation}

Alt, Franz L., Editor

Bartee, Thomas C., Lebow, IRWIN L. \& ReEd, Irving S.

Coulsox, John E.

Fletcher, A., Miller, J. C. P., ROSENhEAD, L. \& Comrie, L. J.
Theory and Design of Digital Machines........... 481
Programmed Learning and Computer-Based Instruction. . . . . ..................... 327 An Index of Mathematical Tables, Second Edition. . 302 


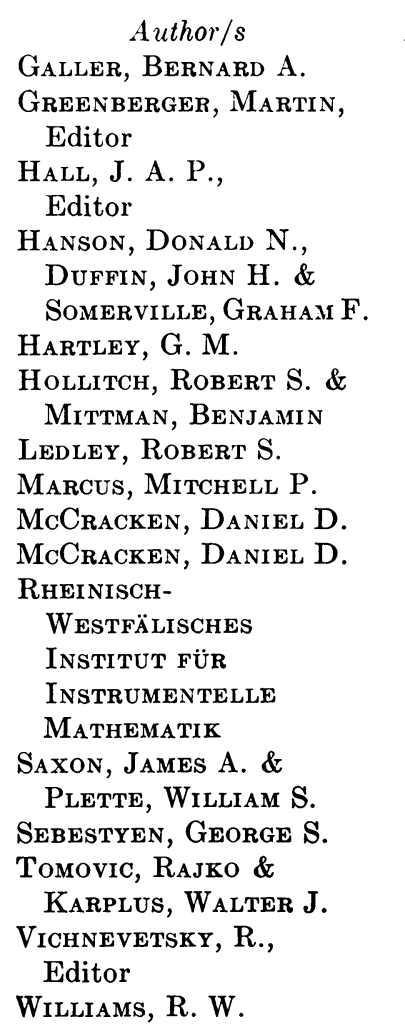

Review Title Page

93 The Language of Computers . . . . . . . . . . . . . . . 482

14 Management and the Computer of the Future....... 97

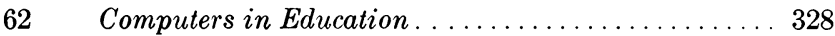

$56 \quad$ Computation of Multistage Separation Processes... . 323

94 An Introduction to Electronic Analog Computers. . . 483

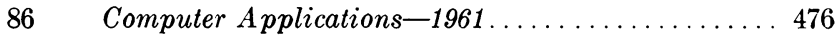

63 Programming and Utilizing Digital Computers. . . . 329

27 Switching Circuits for Engineers................ 208

$64 \quad A$ Guide to $A L G O L$ Programming . .......... 330

$29 \quad A$ Guide to $I B M 1401$ Programming . ........... 210

$30 \quad$ International Series of Numerical Mathematics . . . . 210

65 Programming the IBM 1401: A Self-Instructional

Programmed Manual. ................. 331

91 Decision-Making Processes in Pattern Recognition. . 479

$66 \quad$ High-Speed Analog Computers.............. 331

31 Analogue Computation Applied to the Study of

Chemical Processes...................... 211

32 Analogue Computation................ 213 